\title{
Variations
}

Variations Revue internationale de théorie critique

\section{Parcours non balisés}

\section{Hélène Chatroussat et José Chatroussat}

\section{OpenEdition}

\section{Journals}

Édition électronique

URL : http://journals.openedition.org/variations/247

DOI : 10.4000/variations.247

ISSN : 1968-3960

\section{Éditeur}

Les amis de Variations

\section{Édition imprimée}

Date de publication : 21 décembre 2008

\section{Référence électronique}

Hélène Chatroussat et José Chatroussat, «Parcours non balisés », Variations [En ligne], 12 | 2008, mis en ligne le 01 janvier 2012, consulté le 21 avril 2019. URL : http://journals.openedition.org/ variations/247 ; DOI : 10.4000/variations.247

Ce document a été généré automatiquement le 21 avril 2019.

Les ami•e•s de Variations 


\title{
Parcours non balisés
}

\author{
Hélène Chatroussat et José Chatroussat
}

\section{NOTE DE L'ÉDITEUR}

Première publication sur www.theoriecritique.com, « Wärmestrom, le courant chaud en sciences humaines ", Hiver 2008/2009, pp. 79-96

\section{NOTE DE L'AUTEUR}

Le texte qui suit est un assemblage d'épisodes liés à notre vie et de diverses réflexions sur les forces et les faiblesses du mouvement trotskiste que nous avons connu. Notre optique est subjective. Nous formons un couple depuis longtemps. Nous allons nous exprimer ensemble mais aussi à deux voix distinctes, « Moi, Hélène » ou « Moi, José », par plaisir et par nécessité.

1 Nous avons commencé des études sans les réussir ni les rater complètement. Nous n'avons pas eu un parcours professionnel très classique. Hélène s'est occupée d'enfants en difficulté pendant toute sa carrière, comme institutrice spécialisée et ensuite comme psychomotricienne. José est passé de divers petits boulots à des postes d'instituteur et de professeur de collège et ensuite à un poste de permanent politique qui a pris fin de par sa volonté. Toute notre vie d'adultes s'est déroulée dans l'agglomération rouennaise.

Nous avons milité quelques temps à la Fédération Anarchiste et aux Auberges de Jeunesse, puis trente-deux ans dans le groupe trotskiste " Voix Ouvrière " / "Lutte Ouvrière », et enfin près de trois ans à «Voix des Travailleurs ». Nous avons connu de près et milité aussi avec bon nombre d'autres militants d'extrême gauche, notamment de la LCR. Depuis neuf ans nous ne sommes plus membre d'aucune organisation politique. Nous participons à la revue "Carré rouge " et nous animons le site "Culture et Révolution » depuis septembre 2000 . 


\section{Classifications et singularités}

3 Il y a une expression à Lutte Ouvrière pour caractériser celles et ceux qui ont démissionné ou ont été expulsés comme nous de cette organisation sans pour autant rejoindre la Fraction de LO ou la LCR : « Ils sont dans la nature ». L'expression nous plaît bien car c'est un espace assez large et ouvert pour pouvoir s'exprimer et vivre. Nous serons amenés à dire des choses terre à terre pour rendre visible notre singularité et celle des militants dont nous auront l'occasion de parler.

4 Le monde militant est parcouru par une multitude d'affects et de tensions. Les comportements y sont très diversifiés alors que l'expression des politiques sur le papier ou dans les déclarations publiques semble relativement facile à saisir. Dégager une série de causalités claires et nettes pour rendre compte des réalités du militantisme nous semble plus aventureux.

5 Nous ne dénions pas l'intérêt et la légitimité des études sociologiques cherchant à classer les types d'organisations, les types de pratiques militantes, les types de militants selon l'âge, le sexe, la profession, le niveau de responsabilité dans la hiérarchie du groupe étudié et ainsi de suite.

6 Curieusement ce mode d'appréhension n'est pas dépourvu d'affinités avec la propension à tout classer en catégories et en statistiques développée par le principal dirigeant de LO, Robert Barcia, dit Hardy, avec une efficacité indiscutable. Mais l'efficacité recherchée a changé de terrain progressivement. La « méthodologie organisationnelle » de la direction de LO s'est retournée contre les militants de cette organisation. Elle s'est muée en moyen de contrôle sur eux-mêmes au lieu d'être un appui dans leur intervention. Il y a eu profusion de pratiques artificielles de plus en plus contraignantes. Il n'est plus resté qu'une « gestion du personnel » qui a fini par étouffer complètement les débats vivants et les initiatives stimulantes, et par servir de substitut à une politique digne de ce nom.

7 D'où notre perplexité devant des grilles interprétatives et certaines généralités théoriques ou politiques qui passent à côté des relations militantes tissées (ou déchirées) par des personnes singulières et changeantes.

\section{Quels militants ?}

8 Aujourd'hui nous n'avons toujours pas l'ombre d'un doute quant à la nécessité d'en finir avec le capital comme système destructeur et comme ensemble de relations sociales dans lequel nous sommes immergés. Notre conviction est aussi ardente que lorsque nous avions seize ou dix-sept ans. Mais encore?

Certaines de nos interrogations récurrentes concernent la valeur, les limites et la pertinence aujourd'hui de la figure du militant révolutionnaire et de son intention proclamée de construire le parti ad hoc. Être un militant a de multiples significations souvent contradictoires que nous connaissons par expérience : être à l'écoute des autres et de soi-même, être à l'écoute préférentiellement d'une direction politique, appliquer des schémas et des formules toutes faites, être à la merci de son activisme reposant sur des bases fragiles, se situer dans l'échange égalitaire ou être dans la domination délibéré des autres, être dans la condescendance inconsciente ou non à l'égard de celles et ceux qui ne sont pas des «militants», ou qui sont des militants qui «se trompent» ou qui 
«n'ont pas l'expérience »... Vouloir construire un parti a aussi toutes sortes de significations qui sont largement tributaires de la conception qu'on se fait du militant. S'il se considère comme une sorte d'expert en lutte de classes, on connaît par avance les maigres et tristes résultats d'un tel positionnement.

Nous nous sommes pensés militants d'un futur parti révolutionnaire international, en partie en étant façonnés par un cadre politique déterminé se réclamant du trotskisme, et en grande partie à notre façon. Le projet de construire un tel parti a relevé pour nous de l'évidence pendant quarante ans. Nous ne voulons ni tourner le dos à ce problème ni l'évacuer avec désinvolture. Au-delà de notre cas, il s'agit de reformuler ou de dépasser cette question pour donner plus d'espace, plus de sens et plus d'efficacité percutante aux divers processus d'émancipation. Nous sommes à la fois en position critique vis-à-vis de notre parcours et en point d'appui sur nos expériences et nos convictions initiales.

11 Ce qui pourra apparaître comme rétrospectif ou introspectif dans ce qui suit a en réalité une valeur avant tout prospective pour nous-mêmes et peut-être pour d'autres, pour accomplir quelques pas ensemble vers l'émancipation.

\section{La chute d'Icare}

12 José : Le tableau de Breughel l'Ancien intitulé « la chute d'Icare » représente au premier plan un laboureur en plein effort. On aperçoit au loin en contre bas deux jambes qui s'agitent dans la mer. Ce sont celle du téméraire Icare qui a perdu ses ailes en s'approchant trop du soleil. En 1990, quelques mois après la répression de Tienanmen et la chute du Mur de Berlin, j'ai écrit un petit texte métaphorique pour moi-même car j'avais le pressentiment que de nouvelles difficultés se présentaient dans les relations entre les militants et les travailleurs mais aussi dans leur situation commune dans le monde :

«Il voit s'approcher les eaux froides. Il tombe. Ces eaux atroces, elles sont encore si loin. Mais sa chute est verticale, à la perfection.

Sur la rive transpire le travailleur. Il se concentre sur sa douleur. Il se referme sur son destin gâché. Il ne voit pas le militant-Icare qui a perdu ses ailes. Il ne sait pas encore que son drame est aussi le sien."

13 Etat d'âme ? Quand cela serait. Mais il faut plutôt y voir l'amorce d'une réflexion sur le danger futur que les militants et les prolétaires se retrouvent sur des orbites différentes, que leurs relations s'appauvrissent ou se délitent et que les militants s'enferment dans les simagrées de l'activisme, de la fidélité verbale sans contenu et du renoncement à des recherches et actions authentiquement libératrices. Le moment critique devient alors le refus explicite de glorifier ou de discréditer la figure du militant, tout comme il est celui du refus de glorifier ou de discréditer la figure du prolétaire. Il y a depuis une aspiration à se projeter au-delà et à débarrasser ces deux figures classiques de tout contenu messianique. Notre propre parcours nous y invite.

Nous n'avons jamais vécu notre militantisme en surplomb ou en extériorité aux travailleurs. Ce furent des rencontres nombreuses et ensuite des interventions et des actions où l'échange des savoirs et des émotions était au cœur de nos relations. 


\section{Une ouvrière parmi d'autres}

15 Hélène : Lorsque nous avons commencé à construire un groupe Voix Ouvrière (VO) à Rouen, nous avons fait beaucoup de porte à porte dans les HLM. Cette démarche écartait le préjugé que les militants syndicalistes ou politiques dans une entreprise sont plus conscients a priori que les autres travailleurs. Nous allions donc à la rencontre de travailleurs chez eux, après le travail ou le dimanche matin. On sonnait et on tombait parfois sur un militant du PCF qui nous traitait de tous les noms ou même avait envie de nous casser la figure.

Parfois un travailleur, une travailleuse, une mère de famille acceptait de nous consacrer quelques moments d'échanges précieux. C'est comme cela que nous avons connu Betty au début des années soixante-dix.

Elle travaillait à la chaîne à l'usine Baroclem qui fabriquait de grosses batteries. Elle était une jeune mère de famille et vivait dans un immeuble HLM à moins d'un kilomètre du mien. Elle commentait toutes les grandes et petites crasses de l'exploitation avec une grande force de rire qui réjouissait ses collègues et énervait les chefs (masculins) qu'elles avaient sur le dos. Elle était le pilier du bulletin LO qui paraissait tous les quinze jours sur son usine. Tout y passait sans pitié et avec humour (son humour !) : les mauvais coups des chefs et leurs travers, les bas salaires, les augmentations de cadences, l'hygiène, la sécurité mais aussi la question du respect de chacun. Nous diffusions à la porte mais elle en donnait discrètement à ses meilleures amies. En douce elle en déposait à la machine à café et sur d'autres lieux de passage. Les chefs faisaient une drôle de tête ce qui provoquait des cascades de rires, des sourires entendus ou des airs faussement navrés. Les chefs de la CGT haïssaient ce bulletin qui échappait à leur contrôle. C'était la chasse aux sorcières pour trouver la rédactrice clandestine et lui régler son compte en la dénonçant à la direction. Ce scénario a eu lieu souvent dans les entreprises où Vo et plus tard LO sortait un bulletin.

Pour Betty cela s'est passé autrement. Elle a demandé à être sur les listes de la CGT pour devenir délégué du personnel. Ils ont refusé. Que faire ? Il y avait une grande confiance mutuelle entre nous. Nous avions beaucoup parlé ensemble de tous ses problèmes à l'usine et en dehors. Elle appréciait le respect de ses écrits et le sérieux de notre travail militant. Cela lui a donné le courage d'affronter seule l'appareil CGT de son usine.

avait appris comment le Syndicat Démocratique Renault avait été crée après la grève de 1947. Nous avons puisé à plusieurs reprises dans cette expérience avec nos copains ce qui a abouti notamment à la création du syndicat Démocratie Ouvrière à l'usine Renault-CKD à une époque où les syndicats SUD n'existaient pas.

20 Nous avons suggéré à Betty de faire une campagne de bouche à oreille en faveur du vote blanc au premier tour des élections professionnelles. Le quorum n'a pas été atteint à la consternation des cégétistes et de la direction de l'usine.

21 Comme la direction n'organisait pas un deuxième tour, José est allé voir un inspecteur du Travail. Il a appelé la direction de Baroclem et leur a dit : «Je regrette, c'est la loi, vous êtes obligés. » Au deuxième tour Betty s'est présentée en candidature libre et elle a été élue avec plus de voix que les candidats de la CGT!

Les staliniens étaient en rage. Certains l'ont bousculé dans son atelier. Ils ont même balancé une grosse batterie qui a atterri à quelques centimètres de ses pieds au risque de 
la blesser gravement. Sur les conseils des copains de la direction de LO, on a mis en place un dispositif de dissuasion à l'égard de ces gens-là pour leur passer l'envie d'agresser Betty. Pendant plusieurs matins de suite, entre le domicile du principal responsable de la CGT et l'usine, nous avons placé plusieurs camarades, toujours au même endroit, qui le dévisageaient ostensiblement. Comme par enchantement toute l'équipe des cégétistes a fini par se radoucir à l'égard de Betty. Notre copine a été une très bonne déléguée du personnel, toujours combative et de bonne humeur.

Pour gagner ce genre de luttes, il a toujours fallu que tout fonctionne bien et soit solide. Il fallait avoir gagné la confiance de nombreux travailleurs dans l'entreprise. Il fallait une confiance forte entre les militants impliqués de l'extérieur et ceux qui étaient aux avantpostes et prenaient tous les risques, en particulier celui de se faire licencier et d'être mis sur une liste noire dans la région. Il fallait des personnalités de rebelles voulant aller ensemble jusqu'au bout des possibilités.

\section{LO dans le rétroviseur de VO}

24 Avec un camarade de Nancy qui a milité à LO, à Voix des Travailleurs et à la LCR, nous avons brossé un tableau général de l'histoire parallèle et conjointe de LO et de la LCR depuis les années soixante jusqu'à 2007. [texte intitulé "Où va l'extrême gauche?» téléchargeable sur www.culture.revolution.free.fr ou www.nancy-luttes.net/Rezo-antiK] Nous n'en reprendrons pas ici les éléments. Nous ne comblerons pas non plus les lacunes.

Il semble par contre significatif de mettre en vis-à-vis deux images, deux instantanés, l'un de VO à l'automne de 1965, l'autre de LO en cet automne 2008. Cela pour essayer de repérer les ressorts humains d'une vie collective dans une organisation où s'entremêlent des éléments de continuité et de rupture.

En 1965 le groupe VO parisien qui constituait l'essentiel de cette organisation nous avait beaucoup plu. Les cours marxistes du samedi rue Mouffetard réunissaient tout le monde. Les discussions étaient très animées et se poursuivaient dans la soirée. Il faut imaginer un groupe composé essentiellement de jeunes, en gros de 16 à 25 ans, avec une poignée d'« anciens" du groupe Barta, "l'Union Communiste», ayant un peu plus ou un peu moins de 40 ans. Les plus vieux que nous avons rapidement remarqués étaient Hardy, Vic (Pierre Bois) et Denise. Ils avaient plein de choses passionnantes à raconter, notamment sur la grève de Renault de 1947 dans laquelle le groupe Barta, si petit soit-il, avait joué un rôle fondamental. Nous étions acquis au fait qu'une politique juste est ce qu'il y a de plus important à proposer aux travailleurs. Ils n'avaient pas un comportement de chefs ou de gourous. Ils n'étaient pas toujours d'accord entre eux. C'était une organisation où certains se fâchaient facilement mais pas longtemps. Dans l'ensemble tout le monde riait beaucoup.

Le fait qu'aucun Etat existant ne trouvait grâce dans les analyses de VO nous avait agréablement surpris. Même concernant l'URSS, il était bien entendu pour nos camarades que la meilleure manière de défendre ce qu'il pouvait rester des acquis de la Révolution russe était que les travailleurs renversent la bureaucratie. Les camarades qui faisaient autorité étaient soucieux qu'on ne soit pas méprisants à l'égard des autres groupes même s'ils critiquaient impitoyablement leurs choix militants et leurs analyses. Aucun jeune militant de VO ne pouvait ignorer que ce que nous avions en commun avec les autres groupes trotskistes était précieux. Il était clair que la IVe Internationale ne pourrait être 
reconstruite que par l'ensemble des composantes du mouvement trotskiste Il n'était donc pas question de placer la concurrence sur un mauvais terrain en cherchant à gagner des militants ou des sympathisants déjà en contact avec les autres groupes trotskistes Ces derniers n'avaient pas toujours les mêmes scrupules à notre égard.

En 2008 Lutte Ouvrière nous apparait hagarde, en recherche anxieuse d'une remplaçante à sa porte-parole Arlette Laguiller qui soit crédible médiatiquement, une organisation barricadée dans l'impasse qu'elle a elle-même construite. Sa situation pourrait se résumer par le syllogisme suivant: "Les travailleurs sont démoralisés. Nous sommes liés aux travailleurs. Nous avons donc le devoir d'être démoralisés. Et même d'avantage qu'eux car en tant que militants chevronnés, nous sommes plus conscients de la gravité de la situation. "

Dans la panique de l'entreprise de sauvetage d'une poignée d'élus aux municipales de mars dernier en s'alliant au Parti socialiste, un parti gouvernemental qui a porté de nombreux coups contre les travailleurs, la direction de LO a exclu très logiquement sa Fraction qui protestait contre cette alliance. En l'absence désormais d'opposants internes à stigmatiser, à harceler ou à exclure, un ressort décisif pour maintenir la cohérence du groupe vient de lâcher. Nous laisserons à d'autres les déplorations sur la dérive et l'ossification de LO qui sont effectivement patents.

De la période des années soixante, LO en 2008 a gardé la volonté de ne pas chercher à gagner les militants des autres groupes, de sortir sa presse et ses bulletins en temps et en heure, ce qui était juste et le reste. Mais c'est un peu mince.

\section{Au creux de la nature et de la vie collective}

Hélène : J'étais une enfant turbulente. Mes parents me disaient: "Tiens, tu devrais dessiner cette fleur ou cette plante. » J'aimais cela. Mon père m'emmenait sur le cadre de son vélo découvrir les plantes, les oiseaux, les papillons ou chercher des fossiles dans une marnière. J'étais souvent partie dans la ferme d'en face où j'aidais à ramasser les pommes, à nourrir la volaille et les lapins, où j'ai appris à traire les vaches. Cette passion de la nature ne m'a pas quittée et je l'ai fait partager aussi bien à des enfants qu'à des adultes et en particulier à des militants. J'ai toujours lu des articles et des livres sur la vie des animaux, des livres de biologie, de neurologie. À LO j'ai participé à la réalisation d'un diaporama sur l'origine de la vie. À la fête de LO je me passionnais pour la Cité scientifique qui était réellement vivante au début.

Mes grands-parents, c'était la misère dans un bourg de Picardie. Ils vivaient en grande partie de la récolte de leur jardin. Ma grand-mère était une pailleuse de chaises très expérimentée, payée à la pièce. Mon grand-père avait fait Verdun. Il était ouvrier caviste, très compétent et très mal payé. L'instituteur les a convaincus d'envoyer mon père à l'école normale d'instituteurs. Il y est devenu athée et antimilitariste. Il a rejoint les Jeunesses socialistes de la SFIO d'où il a été exclu, sans réunion ni préavis! Il a rejoint un temps le PSOP de Marceau Pivert. Pendant la guerre il a été envoyé au STO en Allemagne. Il s'est lié à des militants du PCF. Ensuite il a gardé de la sympathie pour certains d'entre eux car il avait été lié à leur réseau de résistance. Mais il a toujours été fermement hostile au stalinisme et nous a toujours soutenus quand les gros bras du PCF nous ont tapés dessus. 

rageur et généreux de Sidney Bechet m'avait envahi comme une vague bienfaitrice. Après ce fut Louis Armstrong, Duke Ellington, Charlie Parker, Miles Davis et bien d'autres. Lorsque le jazz s'est emparé de moi, j'avais 13 ans et j'adorais déjà Schubert, Beethoven et Chopin que, assez curieusement, j'ai alors encore plus aimés. Mais le jazz était une musique autre, «Body and Soul » pour reprendre le titre d'un morceau fameux. Elle était encore largement méprisée de par son caractère sensuel et improvisé. J'entendais des adultes dire que c'était n'importe quoi. Encore heureux quand ils n'affirmaient pas que c'était une musique de sauvages. Je trouvais admirable qu'un peuple opprimé, en butte à un racisme violent et perfide puisse avoir en son sein des musiciens aussi audacieux, avec un comportement décontracté, amusé ou provocateur. En plus ils jouaient avec des musiciens blancs sans aucun préjugé.

Je ne pouvais pas garder la découverte du jazz pour moi seul. Mon meilleur ami prenait des leçons de piano. Après de vives réticences à l'égard de Thelonious Monk qui selon lui jouait faux, il fut conquis. Ensuite il y eut pendant des années un noyau d'amateurs 
passionnés dans notre lycée. Chaque mois apportait son lot d'innovations incroyables. Le jazz cassait la morosité anxieuse de ma vie scolaire.

Les écrivains qui au cours de mon adolescence m'ont le plus aidé à comprendre la vie en société, à supporter cette société tant bien que mal et à la refuser avec constance sont Kafka, Tchekhov, Balzac, Maupassant, Beckett et Boris Vian. Deux œuvres de Kafka m'avaient plus particulièrement sidéré et pénétré profondément: La Métamorphose et Le Château. J'étais fasciné par Olga, celle qui résiste à l'emprise du Château. Je savourais la lucidité ironique de Kafka qui débusque les absurdités étouffantes de la famille et de la société sans en avoir l'air. J'ai retrouvé plus tard cette démarche de contestataire souterrain mais radical chez Melville, dans le personnage de "Bartleby », un employé de bureau qui répète doucement, avec constance, qu'il «aimerait autant pas » (travailler, obéir, se soumettre, être comme tout le monde).

\section{Rencontres marquantes}

José : Je suis d'une famille où il y a des traditions militantes. Mon grand-père qui était receveur des postes était membre actif de la SFIO dans le Nord dès avant 1914. L'affaire Dreyfus l'avait profondément marqué. Mon père n'a pas rejoint la SFIO. Il était antimilitariste, libre penseur, "pacifiste intégral » et "citoyen du monde » comme il disait. Quand il était à l'école normale d'instituteurs de Beauvais, il avait suivi avec d'autres jeunes dans l'arrière-salle d'un café des exposés de Maurice Dommanget, instituteur historien et militant de L'École émancipée, sur l'histoire du mouvement ouvrier.

Mes parents avaient mis en pratique les méthodes pédagogiques de Célestin Freinet quand ils étaient à la campagne. Mon père a milité activement entre autres mouvements à la Ligue des Droits de l'Homme. Il invitait des conférenciers qui mangeaient à la maison. Je me souviens de Lucie Aubrac et de Félicien Challaye, un vieux philosophe pacifiste très bienveillant. Mon père était lié à Louis Lecoin, un anarchiste pacifiste qui avait fait douze ans de prison à cause de ses idées. Il a arraché à De Gaulle un statut pour les objecteurs de conscience en faisant une longue grève de la faim.

Le militantisme de mon père s'accompagnait aussi de soucis que je ressentais avec acuité pendant la guerre d'Algérie. Il avait créé un comité Maurice Audin sur Elbeuf. Il ramenait à la maison des petits livres interdits dont la lecture m'avait beaucoup impressionné : la Question d'Henri Alleg, la Gangrène, « Pour Djamila Bouhired »... Il y avait des calomnies, une ambiance hostile à l'égard de la poignée de gens qui militaient contre la guerre d'Algérie sur l'agglomération. La figure que mon père admirait le plus et sur qui il a fait des conférences et rédigé une plaquette était Francisco Ferrer, un pédagogue anarchiste espagnol fusillé à Barcelone en 1909.

Quand ma mère a commencée comme jeune institutrice, elle s'est retrouvée adjointe de Louis Hobey, un anarcho-syndicaliste qui faisait scandale dans le village. Il avait aidé les ouvriers verriers de la vallée de la Bresle à mettre sur pied leurs syndicats. C'était un anticonformiste, plein de joie de vivre. Il était de santé fragile car il avait eu une enfance misérable et il avait fait toute la guerre de 14 au front. Il en a porté témoignage dans deux livres Un d'en bas et La Guerre? C'est çà ! (éd. La Librairie du Travail). Je l'ai rencontré avec bonheur lorsque j'avais douze ou treize ans dans le village de la Drôme où il avait pris sa retraite. Maintes fois, dans les situations délicates ou pénibles auxquelles mes parents ou lui-même avaient eu à faire face, il répétait avec un rire malicieux : « T’as voulu militer? 
C'est bien fait pour ta gueule... » Militer, c'est un choix, se plaindre n'est pas de mise. À bon entendeur, salut!

\section{La découverte de la musique et son partage} de l'armée française. Un an après l'indépendance, Pagat et ses amis ont décidé d'organiser un camp en Algérie de jeunes Français anticolonialistes et de jeunes Algériens, pour débattre de l'avenir de la révolution algérienne et construire ensemble un lieu d'échanges. Je n'avais pas encore 18 ans mais j'ai décidé d'y aller. Mes parents étaient assez effrayés mais n'ont pas osé s'y opposer. boisés avaient été brûlés par l'armée française. Les règlements de compte armés entre chefs de wilayas continuaient. Au village de Stora, nous nous sommes retrouvés à plus de 200 jeunes, très bien accueillis par la population locale. Il y avait des étudiants de l'UEC, des maoïstes, toutes les variétés de trotskistes, toutes les variétés d'anarchistes. Nous discutions à perte de vue. C'était comme un mini-pré 68. Je me considérais comme anarchiste mais de plus en plus gagné au marxisme.

49 Je me suis lié rapidement à un étudiant lyonnais qui était en contact avec le groupe «Socialisme ou Barbarie». Sur place je donnais des cours dans une école à Skikda le matin et des cours d'alphabétisation l'après-midi. J'ai particulièrement sympathisé avec un jeune pêcheur de mon âge qui avait été maquisard et qui était d'une grande lucidité 
sur ce qui se passait dans son pays. Il m'apprit que le directeur de la conserverie locale était un incapable, un fils à papa d'un ponte du FLN qui n'avait pris aucun risque pendant la guerre et maintenant s'en mettait plein les poches. J'ai discuté avec de jeunes paysans qui rêvaient tous de partir en France pour trouver du travail. Un an seulement après l'indépendance! Le "socialisme algérien» de Ben Bella et compagnie m'apparaissait comme une escroquerie.

De retour à Rouen j'ai eu l'occasion de discuter avec un jeune militant très formé du mouvement trotskiste de Pierre Franck. Il a essayé de me gagner à ses idées. Il pensait que Ben Bella était à la tête d'un "gouvernement ouvrier et paysan " susceptible d'évoluer dans le bon sens. Je lui ai répondu que les ouvriers et paysans algériens avec qui j'avais discuté estimaient que les gens au pouvoir étaient une bande de profiteurs, qui en plus se payaient leur tête avec de beaux discours sur l'autogestion. Je ne l'ai pas ébranlé. Il croyait dur comme fer à cette formule de «gouvernement ouvrier et paysan» tiré du Programme de transition.

\section{De l'anarchisme au trotskisme (allegro vivace)}

51 José : Les années 1963 à 1965 sont pour nous riches en découvertes et rebondissements. Nous militons simultanément aux Auberges de Jeunesse et dans le groupe local de la Fédération Anarchiste (FA). Beaucoup de gens sympathiques et quelques fâcheux dont un couple du PSU qui veulent nous virer du groupe des AJ parce qu'on y fait trop de politique. En hypokhâgne avec un copain anarchiste, nous décidons d'adhérer à l'UEC pour y mettre le bazar. Mon copain est devenu un militant PCF bon teint et je suis parti au bout de trois mois en leur envoyant une lettre de démission dans le genre brûlot.

Nous dévorions toutes les publications d'avant-garde et d'extrême gauche. Nous cherchions notre voie et elles avaient toutes un impact très fort sur nous : l'Internationale Situationniste, Information Correspondance Ouvrière, Front Noir qu'Hélène appréciait particulièrement, Socialisme ou Barbarie et dans une moindre mesure Voix Ouvrière auquel mon père était abonné comme à bien d'autres publications. Je lisais Hegel, Marx, Lukacs, Korsch, Joseph Gabel, Victor Serge, Lénine, Rosa Luxemburg, Trotsky...

Nous avions aussi de bonnes relations avec le groupe des JSU, les jeunes du PSU qui animaient le Cercle Marxiste Rouennais. Ils formeront le noyau initial de la JCR sur Rouen. Lors d'une réunion publique où ils avaient invité Pierre Franck, je lui objectai avec fougue tout l'argumentation de Castoriadis considérant la bureaucratie soviétique comme une classe exploiteuse. Il me répondit très gentiment, sans me convaincre. Au moment où j'allais rejoindre "Socialisme ou Barbarie », ils me firent savoir que le groupe venait de se dissoudre. Faute de mieux, nous continuâmes à la FA.

Aux vacances de 1962 j'avais rencontré au Larzac des anarchistes de la Chaux-de-Fonds à la fois sympathiques et inquiétants dans leurs certitudes bien carrées. J'avais aussi apprécié humainement des militants du PCF ou des gens proches de ce parti en faisant un voyage « Tourisme et Travail » en camping dans plusieurs pays d'Europe avec des familles ouvrières du Havre. L'instituteur responsable de cette équipée avait repéré que je lisais "Le Canard enchaîné ». À la fin il m'avait proposé d'être moniteur et responsable de la trésorerie d'un autre voyage en Allemagne et en Suisse, cette fois avec un groupe d'adolescents pas faciles du tout. Pour rassurer les parents il leur avait fait croire que 
j'avais 18 ans. Le voyage fut assez épique et nous nous sommes très bien entendus pour faire face à tous les problèmes.

Aux vacances suivantes Hélène et moi étions allés en Creuse et l'année suivante en Tchécoslovaquie où sévissait Novotny, pour participer à des chantiers du Service Civil International où nous avions rencontré de jeunes et joyeux contestataires de différents pays. Les jeunes Tchèques avaient un cafard d'encre que Milos Forman a très bien exprimé dans ses premiers films.

56 A la FA nous ne pouvions plus nous contenter de réunions divertissantes et d'actions folkloriques. Toute la faiblesse des écrits de Proudhon et Bakounine et la force de ceux de Marx et de Lénine me sautaient aux yeux. Nous finissions par nous ennuyer du fait que rien n'était entrepris en direction des travailleurs. Nous avons donc quitté le groupe au cours d'une séance assez éprouvante car la plupart ne digéraient pas notre départ, surtout pour rejoindre une organisation trotskiste Seul un vieil anarcho-syndicaliste charmant qui fabriquait des tabliers pour vivre avait compris notre préoccupation de nous lier aux travailleurs et de prendre au sérieux les idées révolutionnaires. J'avais renoué depuis peu avec mon camarade de " Socialisme ou Barbarie » rencontré en Algérie et avec qui après une période de correspondance et des discussions à Rouen, j'avais préféré rompre car je ne comprenais plus ce qu'il me disait. Il faisait partie d'un groupe de jeunes très bohèmes et d'une vaste culture politique qui avaient polémiqué avec Castoriadis au sein de feu "Socialisme ou Barbarie». J'avais entendu le plus influent d'entre eux, Edouard, déclarer: «VO, c'est un groupe bureaucratisé mais ils font du boulot ouvrier sérieux. Qu'est-ce qu'on risque?»

\section{Construire un groupe}

57 José : Il y a une belle nouvelle de Jack London sur un homme cherchant seul à « construire un feu » dans une tempête de neige. Nous avons essayé de construire un groupe Voix Ouvrière à une époque, en 1965-68, où les vents du PCF étaient puissants sur l'agglomération et encore jusqu'au début des années 80 . Nous étions deux au démarrage. Nous étions aidés par des camarades parisiens qui nous donnaient des conseils et surtout nous faisaient découvrir des analyses plus convaincantes que celles des autres groupes. Nous étions particulièrement attachés à Nata, une «ancienne » de 25 ans, qui était très chaleureuse. Elle discutait de nos exposés sur le mouvement ouvrier et nous initiait aux règles organisationnelles de Vo, sans ce formalisme et ce moralisme pénibles qui sévissaient déjà chez certains camarades. Nous avons gagné d'abord nos amis, puis des lycéens, des ouvriers et des employés.

Notre vie n'avait rien de routinière. Sur l'affiche invitant à notre premier meeting, nous avions mis « Pourquoi faut-il construire un nouveau Parti communiste ? » qui a attiré plus de 70 membres du PCF! Des gros bras pour la plupart venu nous expulser et tenir le meeting à notre place, et les responsables et intellectuels locaux du parti venus pour se réjouir du spectacle. Les camarades de la IVe Internationale faisant de l'entrisme dans le PCF avaient eu vent qu'une opération musclée se préparait contre nous. Ils nous avaient prévenus à temps pour que quelques camarades parisiens viennent renforcer notre service d'ordre. L'entrisme « sui generis » a eu réellement du bon en cette occasion. À une vingtaine à peine de jeunots que nous étions, nous avons fait sortir à coups de chaises tous les membres du PCF qui ne se sont pas vantés ensuite d'être venus saboter notre réunion. 
59 A l'échelle du pays les staliniens cherchaient à nous empêcher d'exister, surtout devant les entreprises. À Vénissieux dans la banlieue lyonnaise où se trouvait une grande usine Berliet, c'était l'escalade semaine après semaine. La direction de Vo décida d'envoyer tous ses militants garçons et quelques filles, soit 130 personnes pour protéger nos vendeurs et envoyer un signal fort au PCF qu'ils ne nous bâillonneraient pas. 300 cégétistes déchaînés foncèrent sur nous en nous jetant de grosses pierres à la volée. Nous avions reculé sans perdre notre sang froid et sans leur renvoyer les pierres car il était exclu de prendre le risque de blesser gravement l'un d'entre eux. Nous avons été trois à être hospitalisés. J'avais été traîné à terre et bourré de coups de pieds pendant que les flics arrivés sur les lieux me matraquaient avec entrain dès que je me relevais. À l'hôpital l'hostilité du personnel était glaçante. J'en compris la raison en rentrant à Rouen. La presse, avec une touchante unanimité, nous avait présenté comme un commando de maoïstes ayant agressé de paisibles ouvriers de chez Berliet.

J'avais été encore embarqué par la police pour quelques heures en juillet 68 à la suite d'une diffusion devant Renault-Cléon. Vo était un des dix groupes qui venaient d'être interdits et les flics étaient plutôt nerveux à notre égard. J'eus l'occasion de refaire un petit séjour à l'hôpital à Rouen après avoir été agressé lors d'un collage par un petit commando d'extrême-droite armé de nunchakus. Des individus du même genre avaient commis une opération beaucoup plus conséquente avant 68 sur le campus de Rouen faisant un blessé grave parmi les camarades de la JCR. La solidarité a toujours bien joué entre nous face aux gros bras du PCF qui empêchèrent aussi physiquement la tenue d'une réunion publique JCR en l'honneur de Che Guevara, face aux nazillons en diverses occasions ou face aux flics quand nous avons manifesté ensemble contre la dictature franquiste.

\section{Mettre en pratique ses idées}

61 Hélène : Ma façon de gagner les gens passait par des relations personnelles. Des liens d'amitié. À la vie, à la mort. Je disais aux gens que je voyais, j'ai fait un choix de vie. Je leur expliquais mes idées et souvent je les ai gagnés à mes idées. Dans mon travail d'institutrice spécialisée, il y avait un engagement du même ordre. J'ai suivi un chemin où j'ai mis en pratique mes idées de fraternité, d'empathie, d'être avec les autres.

José : La façon de gagner les gens n'étaient pas encore codifiée à notre niveau rouennais. Le problème c'était de gagner sur le fond : « La révolution mondiale. Changer la société de fond en comble sur l'ensemble de la planète. Voilà l'enjeu. Une société communiste, sans États, sans oppression. Comment faire? Quelle est la force qui peut réaliser ça ? La classe ouvrière, partout, dans tous les pays. C'est elle qui va porter la culture humaine.» On n'inventait pas la poudre. Mais le problème c'était d'y croire, de le faire partager. Et par voie de conséquence de convaincre de rejoindre le groupe qui propose le mieux de défendre ces idées-là au sein de la force motrice, le prolétariat. À nos yeux cela impliquait des liens particuliers entre nous et avec les gens. Ça faisait un ensemble. Nous voulions une société communiste à l'échelle mondiale. La moindre des choses était d'établir entre nous et autour de nous des relations fraternelles en toutes circonstances, à l'image de la société que nous voulions. 


\section{Pas tout à fait dans la norme}

63 mettre en congé pour faire un stage de formation VO. Au risque de se faire mal voir de ses
collègues et de compromettre son travail. Quitter trop souvent mon travail dans l'enfance inadaptée me posait un problème, pas de culpabilité, mais d'implication vis-à-vis des enfants. Parce que j'étais aussi largement engagée envers eux. Au moins autant que vis-àvis de mes idées ou des copains de mon organisation. Ces mômes, j'avais des comptes à leur rendre. Les responsables de VO n'étaient pas d'accord pour que je fasse ce métier mais j'ai persisté, pendant 30 ans, parce que j'ai une tête de mule. Dans mon travail, j'ai tenu tête aux inspecteurs de la même façon. J'étais convoquée, engueulée. Mes collègues et moi, nous étions contre les tests et les QI. Je n'ai jamais été comme il fallait dans l'enfance inadaptée. Je résistais à l'administration.

64 m'avait dit, «je te paye tes études, mais tu ne redoubleras pas ». J'ai redoublé une fois. Alors il ne me restait plus qu'à être institutrice. Pourquoi pas dans l'enfance inadaptée puisque je n'avais pas pu faire médecine comme j'en avais rêvé?

José : VO s'est incliné de mauvaise grâce devant le fait accompli, le double militantisme d'Hélène. Elle avait gagné beaucoup de gens, des étudiants, des lycéens et des salariés. Difficile de l'écarter mais il ne fallait pas que ça soit un exemple à suivre.

Peu importe ce reproche, nous avions un enthousiasme très fort. Mais nous avions bien remarqué que nous n'étions pas tout à fait dans la norme. Hardy avait dit dans un des premiers stages que pour construire un groupe, ça ne peut pas se faire à partir d'un couple. Considération organisationnelle de fond ! C'est embêtant, parce que nous, on était un couple. On a démarré et continué comme ça. Il y avait un message derrière. Cela aurait été plus objectif, plus politique, si on s'était séparé. Tout en continuant à militer et à être en bons termes bien sûr. Un couple, ça peut être une entité très résistante aux pressions de la famille mais aussi à celles d'un groupe politique. Les tentatives infructueuses de briser notre couple n'ont pas manquées, y compris beaucoup plus tard à Voix des Travailleurs.

67 À LO, nous aurons amplement l'occasion dans les années ultérieures de découvrir à nos dépens les arcanes de la lutte contre les tendances « individualistes petites bourgeoises ».

\section{Service militaire}

José : Au début des années 70 notre confiance dans l'organisation n'était déjà plus sans failles. Les effectifs progressaient mais l'autoritarisme et le moralisme aussi, sous couvert de se prémunir du gauchisme et de ses modes.

Les responsables de LO m'avaient proposé un tuyau pour me faire réformer étant donné ma situation de responsable du groupe Rouen. J'ai décliné la proposition car je ne voulais rien leur devoir. Dans mon for intérieur, je ne trouvais pas juste d'échapper au service militaire alors que les jeunes des milieux populaires ne disposaient pas eux de combines. J'ai donc fait mon service d'août 72 à juillet 73 dans un régiment d'infanterie semidisciplinaire, en étant en plus mal vu par mon organisation qui m'a battu froid pendant ces douze mois. 
70 Les adversaires les plus redoutables au service militaire étaient la betise, l'ennui et les périodes de manœuvres où les gradés étaient très excités. Plus des trois-quarts des appelés venaient de milieux populaires. Certains avaient déjà fait de la prison pour « des conneries ». Il ne fallait pas leur marcher sur les pieds ni leur faire de grands discours de morale ou de politique.

71 J'ai essayé de donner envie de faire certaines choses sans chercher à convaincre. Je lisais un roman et un gars jetait un œil. «Si tu veux, je te le passe, tu verras bien si ça te plaît. » Je jouais beaucoup aux échecs avec un militant du PCF. À certains gars je disais : « Je peux t'apprendre la règle si tu as envie.» Résultat un certain nombre de gars qui étaient ouvriers chez Renault ou ouvriers agricoles finirent par « pousser le bois » comme dit Le Neveu de Rameau. Au cours d'une manœuvre je décidais d'arrêter de fumer définitivement. Quelques copains de la chambrée m'emboîtèrent le pas avec succès, d'autant plus que c'était pour provoquer l'armée qui de fait nous mettait aussi sous sa dépendance en encourageant le tabagisme et l'alcoolisme.

72 Nous avions dû monter à Paris trois jours pour préparer le défilé du 14 juillet. Sur un temps de quartier libre nous sommes allés visiter quelques galeries du Louvre que je connaissais bien et tout le monde bichait. Les prolos étaient là où on ne les attendait pas et où ils ne s'attendaient pas à aller. Vers la fin du service je déclarais sans insister : «Faites comme vous voulez. Moi, je ne vais pas me saouler pour la quille et d'ailleurs je ne vais pas acheter une quille en bois. " Tout le monde a été d'accord, y compris les gros consommateurs de bière. La fin du service a été d'un calme inquiétant pour les gradés.

73 Je ne peux pas dire que j'ai fait un «travail politique » à l'armée mais j'ai appris beaucoup de mes compagnons de chambrée.

\section{Infraction aux « principes »}

74 Hélène : Il était interdit d'avoir un enfant à LO. Il paraît que ça ne l'est plus. Nous n'avions pas choisi d'avoir un enfant mais comme presque toutes les femmes, je me suis trouvée enceinte. José était à l'armée. Quand je l'ai annoncé à une militante parisienne qui descendait à Rouen à cette époque pour suivre ce que faisait notre groupe, elle est montée sur ses grands chevaux. Je devais renoncer à cette maternité car non seulement je ne pourrais pas militer mais cela empêcherait aussi José de le faire. Elle était très agressive. J'ai tenu tête et je l'ai envoyée sur les roses. Quelques temps plus tard j'ai perdu cet enfant.

75 José : Nous comprenions très bien qu'il fallait se rendre le plus disponible possible ce qui impliquait de faire le choix de ne pas avoir d'enfant. Mais trop souvent à LO, il ne s'agissait pas de présenter un choix et de convaincre mais d'exercer des pressions morales parfaitement odieuses. On fit également pression sur moi : je devais convaincre Hélène de se faire avorter. Nous n'avions pas besoin de leurs « leçons » pour savoir ce que nous voulions décider tous les deux. Leur céder me semblait pire que tout sur tous les plans. Je m'étais fait à l'idée d'être rétrogradé comme militant de base ou sympathisant.

À mon retour du service militaire, une crise éclata dans notre groupe local. Nous l'avons surmonté en déployant un surcroît d'énergie. Être en position de «dirigeant» me déplaisait plus que jamais. Je n'ai jamais su me comporter en « responsable» remettant les uns vertement à leur place et se laissant agréablement flatté par les autres. Ces délices de l'exercice du pouvoir même à petite échelle m'ont toujours déplu. Mais l'actualité des 
grèves et des mouvements des jeunes des collèges techniques dans lesquels on jouait un rôle m'avait persuadé que ce n'était pas du tout le moment de me mettre en retrait. Un jour, les dirigeants de l'organisation me proposèrent, à ma grande surprise, d'assister aux réunions hebdomadaires du Comité exécutif (CE). Une position peu enviable mais idéale pour comprendre l'évolution de LO, de sa politique et de la qualité des relations humaines entre militants.

\section{De l'humour, de la bonne humeur, de l'estime de soi et des autres}

Pour apprécier la qualité de la vie intérieure à une organisation politique se voulant révolutionnaire, il est décisif d'y déceler la présence ou l'absence d'humour et de bonne humeur car cela n'affecte pas seulement les relations entre militants mais aussi celles avec les gens qui ne militent pas. Il faut prendre ici l'humour dans sa dimension collective, comme faculté de rire de soi et des autres, comme une modalité particulière pour un groupe de porter utilement la critique sur sa politique et ses pratiques tout autant que sur celle des autres.

Il n'y a pas eu une époque bénie où l'humour régnait sans partage. Mais il faut bien constater que la courbe est plutôt descendante depuis plus de quarante ans.

Ces critères de l'humour et de la bonne humeur collective sont en fait décisifs pour la création, le développement et la viabilité d'un projet politique, qu'il s'agisse d'éditer par exemple une revue ou de vouloir construire un «nouveau parti anticapitaliste ». Il n'y a pas de recette pour faire fleurir cela, mais une des conditions primordiale est l'établissement de relations égalitaires où prévaut constamment la raisonnable estime de soi et des autres. En d'autres termes tous les phénomènes d'autoritarisme et d'hystérie et leurs corollaires de la servitude volontaire ou de la dévalorisation de soi ont rendu bien des groupes et bien des entreprises politiques non seulement ennuyeuses ou repoussantes pour bien des militants, mais évidemment fortement dissuasives pour les personnes qui en principe auraient pu s'y impliquer. Somme toute, l'humour est quelque chose qu'il faudrait prendre au sérieux.

À suivre...

\section{AUTEURS}

\section{HÉLÈNE CHATROUSSAT}

Du comité de rédaction de Variations

JOSÉ CHATROUSSAT

Du comité de rédaction de Variations 